\title{
A prospective comparative study of functional outcome following arthroscopic debridement and hydrocortisone injection vs arthroscopic debridement and hyaluronicacid injection for the treatment of early primary osteoarthritis knee
}

\author{
Vaibhav Patel ${ }^{1}$, Neil Rohra ${ }^{2 *}$ \\ ${ }^{1}$ Senior Resident, ${ }^{2}$ Assistant Professor, ${ }^{1}$ GMERS Medical College, Gandhinagar, Gujarat, ${ }^{2}$ GMERS Medical College, Himmatnagar, \\ Gujarat, India
}

*Corresponding Author: Neil Rohra

Email: drneilrohra@gmail.com

\begin{abstract}
Objective: Early knee osteoarthritis in all cases, lead to degenerative medial meniscus lesions which are visible in MRI as chondral defects. As a result, it is difficult to distinguish between unstable meniscus and early osteoarthritis which leads to symptomatic knee pain. Hence, we decided to study improvement athroscopic meniscectomy and injecting Hyaluronic acid or Hydrocortisone in cases of degenerative ruptures of the medial meniscus with changes of early stage medial compartment knee osteoarthritis.

Materials and Methods: 60 consecutive cases of early OA Knee of non traumatic origin were studied. They were given treatment of arthroscopic lavage and randomly administered hydrocortisone or hyaluronic acid. The WOMAC score was obtained and compared from immediate post-op period to upto 6 months.

Result: In our study on patient undergoing arthroscopic debridement of osteoarthritis knee with Inj. Hydrocortisone improvement in WOMAC score was from $50.97 \%$ to $43.81 \%$, totally an improvement of WOMAC score $7.2 \%$ while those undergoing arthroscopic debridement of osteoarthritis knee with Inj. Hyaluronic acid improvement in WOMAC score was from $51.25 \%$ to $37.1 \%$, totally an improvement in WOMAC score of around $15 \%$.

Conclusion: Joint lavage by arthroscopy in combination with either intra-articular steroid injection or intra-articular hyaluronic acid injection provides significant improvement in osteoarthritis knee patient. Data suggests that, those patient who underwent arthroscopic debridement and injection of hydrocortisone had symptomatic improvement at early follow up more than those of the hyaluronic acid, while arthroscopic debridement of osteoarthritis knee and injection of hyaluronic acid performed better at longer follow up.
\end{abstract}

Keywords: WOMAC score, Hyaluronan, Osteoarthritis.

\section{Introduction}

Osteoarthritis is defined as a degenerative, non-inflammatory joint disease characterized by destruction of articular cartilage of the joint and new bone formation at the joint surfaces and margins. ${ }^{1}$ The term osteoarthritis was first coined by John Spendon. However, it can be considered a misnomer and the right term is Osteoarthrosis or degenerative joint disease. ${ }^{2}$ Osteoarthritis (OA) is a common noninflammatory condition, found in $80 \%$ population radiologicaly over the age of 40 . It affects most commonly the knee joint, followed by the hip joint. Patients main complain will be pain. ${ }^{3}$ Osteoarthritis is characterised by wearing of articular cartilage with subsequent damage to the bones, synovial membrane and meniscus. Osteoarthritis causes joint pain, chronic effusion, osteophytes formation around the joint, subchondral sclerosis and cyst formation or meniscal tears. ${ }^{4}$ Osteoarthritis is a clinical syndrome wherein patient has joint pain accompanied by varying degrees of functional limitation leading to decrease in quality of life. ${ }^{5}$ It affects several aspect of a patient's life including, social activities, functional activity and relationships and emotional well being. ${ }^{6}$ Management of patients with OA can either be done conservatively that includes lifestyle modifications, medications such as non-steroidal anti-inflammatory drugs, and physical therapy or surgically, which is indicated when conservative treatment fails. Surgical options include nonarthroscopic and arthroscopic lavage, arthroscopic debridement, corrective osteotomy, and total knee replacement. Arthroscopic lavage with debridement is frequently used in a treatment of painful degenerative knee which relieves the symptoms. Both open and arthroscopic lavage are commonly used techniques, consisting of washing out the knee with around 10 litres of fluid. Any intra-articular debris, including microscopic and macroscopic cartilage fragments or calcium phosphate crystals which may cause pain or inflammation, are cleaned and flushed out using shaver and cannulas. ${ }^{7}$ Osteoarthritis is the most common cause of long term disability next in frequency to chronic heart disease. In adult age group prevalence is around $16 \%$ in women and around $25 \%$ in men. ${ }^{8}$ Movement limitation is found in more than $80 \%$ of patient with OA while around $25 \%$ cannot perform activities of daily life. ${ }^{9}$ Osteoarthritis prevalence increases with age. Below 45 years it's more common in men, while over the age of 45 years it's more common in women. ${ }^{10}$

Initial reports of arthroscopic treatment in cases of early arthritic knee originate in the 1920 s which were reported by Bircher describing the beneficial effects of diagnostic arthroscopy. ${ }^{11}$ Thereafter Burman et al. also reported use of arthroscopic lavage in cases of early OA knee in 10 patients 1930s, they reported significant improvement in almost all patients. ${ }^{12}$ Watanabe et al. had used arthroscopic lavage in reducing symptoms of osteoarthritis extensively in the 1950s. ${ }^{13}$ It was following the experimentation of Magnusun and Pridie, in which open debridement of the knee was done, interest arose amongst researchers for exploring arthroscopic 
debridement in early OA knee and marrow stimulation mainly due to advancement of instrumentation of arthroscopy in 1970s. Diagnostic Arthroscopy became an important tool for the assessment of the extent of cartilage injury, to determine the need for either osteotomy or prosthetic replacements in the patients. ${ }^{14}$ In the past decade, large number research articles have been devoted to further define indications for arthroscopic interventions for osteoarthritis because of economic pressures and the fear that arthroscopy is overused. ${ }^{15}$

Injection of Cortisone in the treatment of muscle and joint inflammatory conditions is now a days quiet popular. It was first popularized by Janet Travell MD, who observed that muscle injections of cortisone have remarkable effect and act as adjunct to drugs and physical therapies and are also safe and quite easy to perform. ${ }^{16}$ It is technically more difficult to perform joint injections, but are greatly helpful in patients symptomatic relief. Steroids are quite similar to substances produced by the body (hormones) that help reduce inflammation. Steroids also help in reducing inflammation in tendons and ligaments surrounding the osteoarthritic joints. Use of injectectable corticosteroid in the joint can be done in patients who donot have relief by treatment using analgesics or non steroidal anti-inflammatory drugs. The relief obtained could last from a few weeks to around 6 months. ${ }^{17}$ The mechanism of corticosteroid is reduction in inflammatory reaction by decreasing capillary dilatation and reduces permeability of the vascular structures. These steroids also restrict the accumulation of polymorphonuclear leukocytes and macrophages and thereby reducing the release of vasoactive kinins. ${ }^{18}$ Newer studies have also suggested that corticosteroids help in inhibiting release of arachidonic acid from phospholipids, thereby reducing the formation of prostaglandins, prostagladins enhances the inflammatory process. ${ }^{19}$

Hyaluronic acid (HA) that is present in high levels in the synovial fluid which is a major component of the extracellular matrix of cartilage and superficial layers of the synovial membrane. It helps in buffering the joint load transmission, joints lubrication and imparting antiinflammatory properties to synovial fluid. ${ }^{20}$ Within the articular environment, HA plays a dominant role in maintaining the functional and structural properties of the cartilage matrix, synovial fluids viscoelastic properties and in the regulation of cellular activity via interaction of specific HA cellular receptors and HA glycoprotein. ${ }^{21}$ Several in vitro and in vivo studies have shown that HA induces proteoglycan synthesis and aggregation, ${ }^{22}$ stimulating production of HA within, ${ }^{23}$ modulation of the inflammatory responses, ${ }^{24}$ reduction of chemotaxis and leucocyte, neutrophils, lymphocytes migration, ${ }^{25}$ and also in scavenging effects on free oxygen radicals ${ }^{26}$ induced breakdown of cartilage. In osteoarthritis the molecular weight and concentration of hyaluronan is reduced, resulting in a reduction in the viscoelasticity of the fluid and in the increased susceptibility to wear. In arthroscopic debridement with intraarticular injection of hyaluronic acid the pathologic osteoarthritis synovial fluid is removed and replaced by hyaluronan based products that restore the molecular weight and concentration of hyaluronan to normal values. Previously various clinical trials have tested the efficacy of Inj. HA induced symptomatic benefits in the treatment of OA. ${ }^{27}$ Multiple experimental studies have suggested that HA may influence the cartilage structure and chondrocyte vitality preservation, as well as in reducing synovial cell proliferation that inturn has influence on the degenerative process as a whole. ${ }^{27}$

\section{Materials and Methods}

After obtaining clearance from local ethical committee, 60 consecutive patients of age up to 60 years, with primary Osteoarthritis Knee, outerbride grade 2 and 3 were selected for study. This was a prospective randomized control study and the patients were randomly divided into 2 groups. Group HA received arthroscopic debridement and hyaluronic acid while Group HC received arthroscopic debridement and hydrocortisone as treatment. Out of 60 patients, 36 patients were female and 24 patients were male. So the incidence was significantly higher in Female. The female and male ratio is $3: 2$. In order to compare the two means, data were analyzed using both descriptive statistics and unpaired t-test procedure. Processing was done using GraphPad Prism 6 (GraphPad Software, Inc).

Total number of Grade 2 Outer bridge patients were 32, among them 20 were female patient and 12 were male patient. While total number of Grade 3 Patients were 28, among them 16 were female patient and 12 were male patient.

\section{Results}

The results of WOMAC scores of all the patients studied is tabulated below:

Table 1

\begin{tabular}{|l|c|c|c|c|c|}
\hline $\mathbf{n}=\mathbf{6 0}$ & Pre Ope & Post Ope & 6 Week & 12 Week & 6 Month \\
\hline Inj.HC \& WOMAC Mean & 48.93 & 34.53 & 32.93 & 39.13 & 42.06 \\
\hline Inj.HA \& WOMAC Mean & 49.2 & 39.13 & 39.06 & 36.06 & 35.6 \\
\hline
\end{tabular}

Follow up result of WOMAC score 
Table 2

\begin{tabular}{|l|c|c|c|c|c|}
\hline \multirow{2}{*}{ Post-operative } & Group & Mean Variance & Std. dev & t-test & P value< $<0.05)$ \\
\cline { 2 - 5 } & Inj.HC & 6.981 & 2.6422 & 15.5248 & \multirow{2}{*}{ Highly Significant } \\
\cline { 2 - 5 } 6 Weeks & Inj.HA & 3.6952 & 1.9223 & 13.0937 & \\
\cline { 2 - 5 } & Inj.HC & 4.781 & 2.1865 & 18.94 & \multirow{2}{*}{ Highly Significant } \\
\cline { 2 - 5 } 3 Month & Inj.HA & 12.9238 & 3.595 & 9.2261 & \\
\cline { 2 - 5 } & Inj.HC & 5.981 & 2.4456 & 11.0002 & \multirow{2}{*}{ Highly Significant } \\
\cline { 2 - 5 } 6 Month & Inj.HA & 13.3524 & 3.6541 & 11.8185 & \\
\cline { 2 - 5 } & Inj.HC & 7.781 & 2.7894 & 7.1838 & \multirow{2}{*}{ Highly Significant } \\
\cline { 2 - 5 } & Inj.HA & 9.6857 & 3.1122 & 13.6654 & \\
\hline
\end{tabular}

\section{Discussion}

In our study we had $43 \%$ Outerbridge grade 2 patient and $57 \%$ grade 3 patient, and female and male ratio was $3: 2$. So in our study female preponderance was more than male. Prevalence of grade 3 was also observed more in study of $\mathrm{L}$. Frrizziro, E.Govani and P.Bacchini ${ }^{28}$ in 1998.

In our study the mean age was 51.37 years including both female and male population, ranging from 40-60.In multiple international study studies mean age was around $60 .{ }^{29-32}$ In Indian study by Sharma N.R. et al; mean age group was $51.28^{29}$ which are similar to our observation. This could be attributed to earlier occurrence of osteoarthritis knee in Indian subcontinent due to Indian culture and habits.

In our study we had divided patient equally into those receiving Inj.Hydrocortisone and Inj.Hyaluronic acid after arthroscopic debridement of early osteoarthritis knee. In our study we used WOMAC score for functional outcome with osteoarthritis knee addressing knee pain, stiffness and physical function as it is considered important tool for identification, gradation and management of osteoarthritis knee patients. ${ }^{29}$

In our study, on patients who underwent arthroscopic debridement of osteoarthritis knee with Inj.Hydrocortisone improvement in WOMAC score was from $50.97 \%$ to $43.81 \%$, totally an improvement of WOMAC score $7.2 \%$. Similar improvement in WOMAC score of $7.8 \%$ found by Smith M.D. et $\mathrm{al}^{30}$ in a population of 71 patients to assess the outcome of intra articular steroid injections following arthroscopy and joint lavage in symptomatic osteoarthritis of the knee.

In our study on patient undergoing arthroscopic debridement of osteoarthritis knee with Inj.Hyaluronic acid improvement in WOMAC score was from $51.25 \%$ to $37.1 \%$, totally an improvement in WOMAC Score of around $15 \%$. Similar improvement in WOMAC score of $18 \%$ found by Xinning Li et $\mathrm{al}^{31}$ in a population of 30 patients. While a study by Dervin et $\mathrm{al}^{32}$ showing effects of arthroscopic debridement for osteoarthritis knee showed an improvement of $15 \%$ in patient treated arthroscopically.

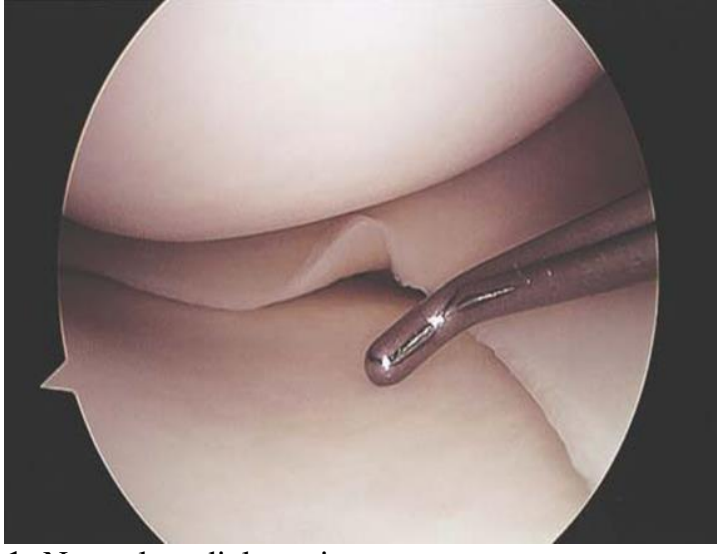

Fig. 1: Normal medial meniscus

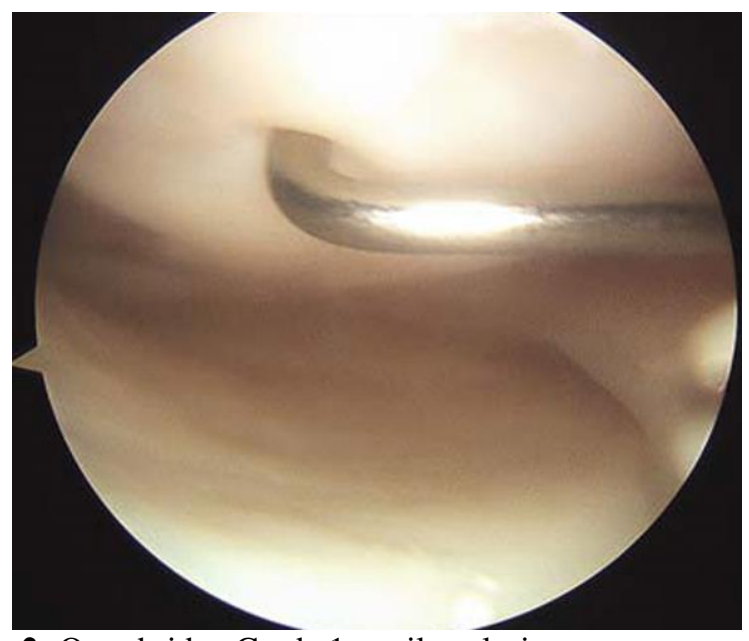

Fig. 2: Outerbridge Grade 1 cartilage lesion 


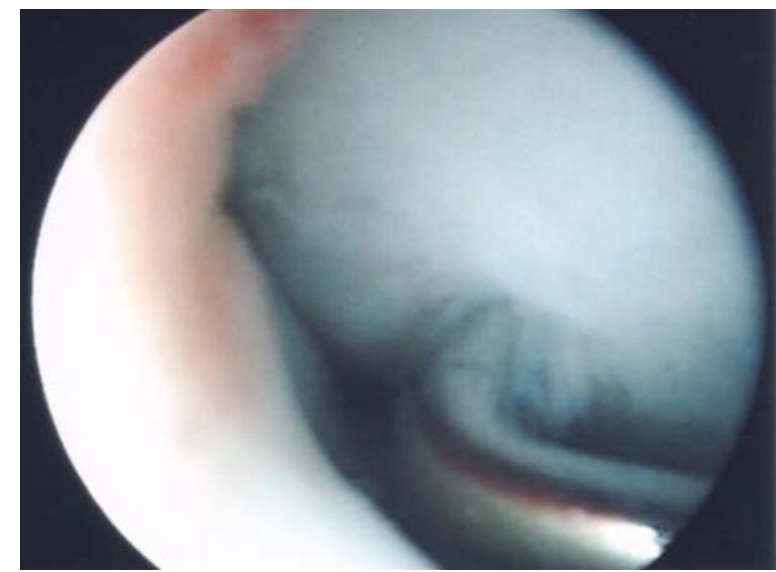

Fig. 3: Outerbridge grade 2 lesion of lateral femoral condyle

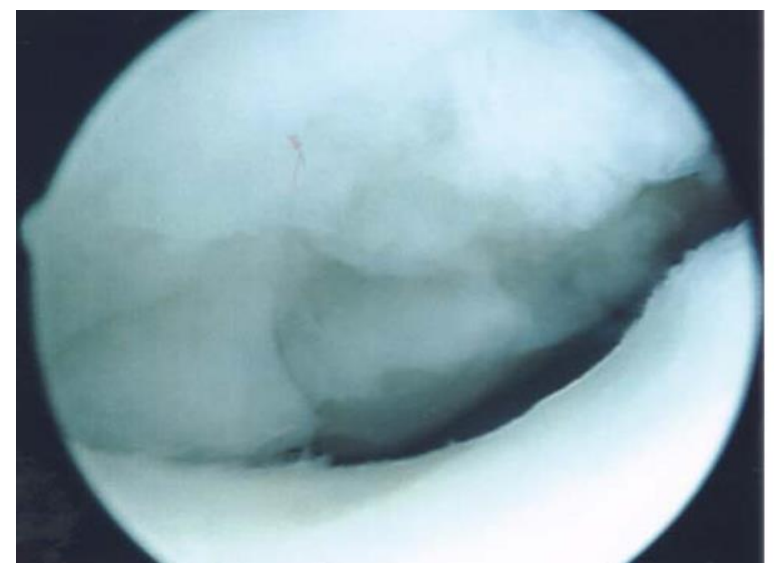

Fig. 4: Outerbridge grade 3 lesion of patella

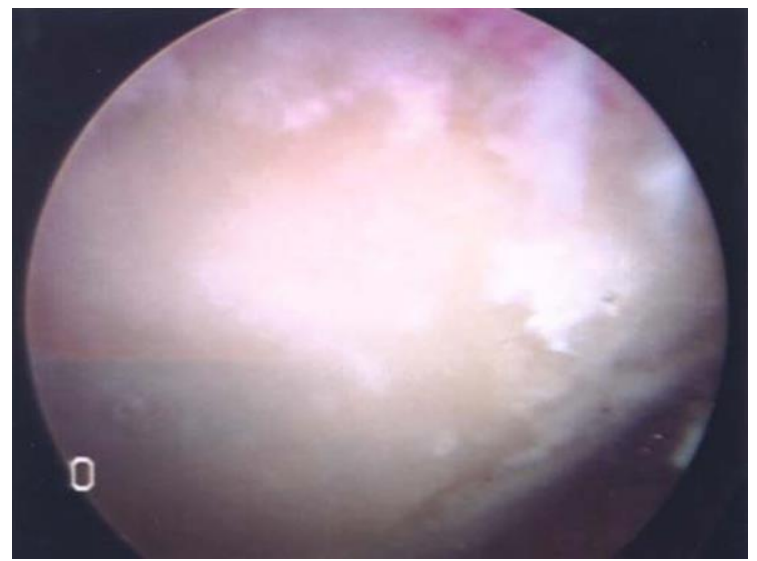

Fig. 5: Outerbridge grade 4 lesion of medial femoral condyle

In our study there was stastically significant improvement in WOMAC score $(\mathrm{p}<0.00001)$ between pre operative and at each follow up for both group of patients treated with Injection hydrocortisone and Injection hyaluronic acid following arthroscopic debridement of osteoarthritis knee. Similar stastically significant improvement in WOMAC scores were observed study by Smith M.D. et al. ${ }^{30}$ for patients treated with intra-articular steroid injection following arthroscopy and joint lavage in symptomatic osteoarthritis knee.

There are several limitations in our study. It is a case series, there were no control groups, and we had short follow up of 6 month with the sample size of 30. However, the strength of study is that all procedure was performed by single surgeon and study is prospective study. To our knowledge it is a first case series comparing Injection hydocortisone VS Injection hyaluronic acid following arthroscopic debridement of knee utilising WOMAC score.

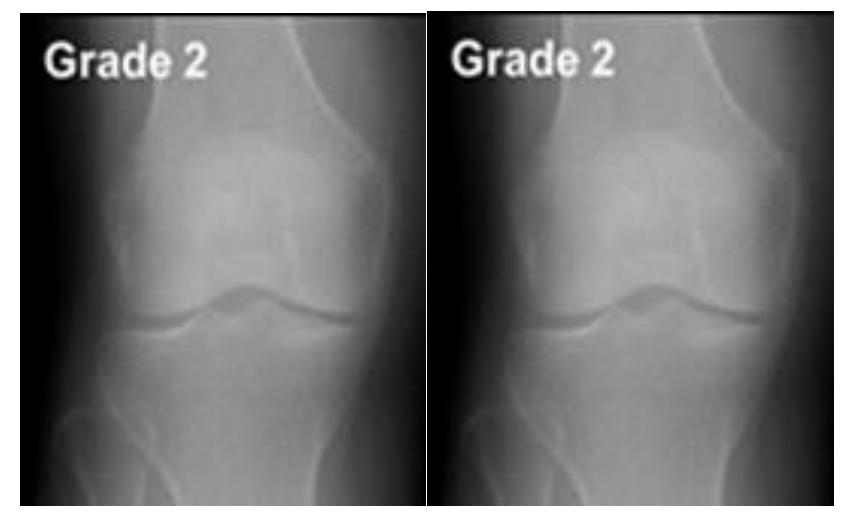

Fig. 6: Pre-op and post-op standing x-ray of patient with outerbridge grade 1 lesion

\section{Conclusion}

Joint lavage by arthroscopy in combination with either intraarticular steroid injection or intra-articular hyaluronic acid injection provides significant improvement in osteoarthritis knee patient. These preliminary data suggests that, patient who underwent arthroscopic debridement of early osteoarthritis knee and injection of hydrocortisone had better symptomatic improvement at early follow up while arthroscopic debridement of osteoarthritis knee and injection of hyaluronic acid performed better at longer follow up. Both hydrocortisone and hyaluronic acid injection were found to be safe and complication free. These benefits were more significant in patients with mild to moderate osteoarthritis knee.

\section{Conflict of Interest: None.}

\section{References}

1. Ebnezer J, "Osteoarthritis; Definition of osteoarthritis" Chapter 47 in John Ebnezer Text book of Orthopaedics, $4^{\text {th }}$ edition 2010, pp.674

2. The Cochrane Collabration Journal. Bellamy N, Campbell J, Welch V, Gee TL, Bourne R, Wells GA, 2006;20:5-6.

3. Dandy DJ. Editorial - Arthroscopic debridement of the knee in Osteoarthritis. JBJS [Br] 1991;73:877-8.

4. Burks Rt. Arthroscopy and degenerative arthritis of the knee: a review of literature, Arthroscopy. 1990;6:43-7.

5. ACR (2000) Recommendations for the medical management of osteoarthritis of the hip and knee: 2000 update. American College of Rheumatology Subcommittee on Osteoarthritis Guidelines. Arthritis Rheum 43(9):1905-15.

6. Carr AJ: Beyond Disability: measuring the social and personal consequences of osteoarthritis. Osteoarthritis Cartilage 1999:7:230-8. 
7. Bradley JD, Heilman DK, Katz BP, Gsell P, Wallick JE, Brandt KD. Tidal irrigation as treatment for knee osteoarthritis: a shamcontrolled, randomized, doubleblinded evaluation. Arthritis Rheumatism 2002;46(1):100-8.

8. Hochberg MC. Role of intra-articular hyaluronic acid preparations in medical management of osteoarthritis of the knee. Semin Arthritis Rheum 2000;30(2 Suppl 1):2-10.

9. Karlsson J, Sjogren LS, Lohmander LS. Comparison of two hyaluronan drugs and placebo in patients with knee osteoarthritis. A controlled, randomized, double-blind, paralleldesign multicentre study. Rheumatology 2002;41(11):1240-8.

10. Altman RD. Osteoarthritis, Differentiation from rheumatoid arthritis, causes of pain, treatment. Postgrad Med 1990;87(3):66-72.

11. Bircher E. Die Arthroendoskopie. Zentralbl Chir 1921;48:1460-1.

12. Burman MS, Finkelstein H, Mayer L. Arthroscopy of the knee joint. J Bone Joint Surg 1934;16:25.

13. Watanabe M, Takeda S, Ikeuchi H. Atlas of Arthroscopy. Tokyo: Igaku Shoin, 1957.

14. Lysholm J, Hamberg P, Gilquist J. The correlation between osteoarthritis as seen on radiographs and arthroscopy. Arthrosc 1987;3:161-168.

15. Jackson RW. The role of arthroscopy in the management of disorders of the knee. Clin Orthop 1974;101:28-35.

16. Miller JH, White J, Norton TH. The value of intra-articular injections in osteoarthritis of the knee. J Bone Joint Surg Am 1958;40A:636-43.

17. Fridman DM, Moore ME. The efficacy of intra-articular steroids in OA; a double blind study. J Rheumatol 1980;7:8506.

18. Dieppe PA, Sathapatayavongs B, Jones HE, Bacon PA, Ring EFJ. Intra-articular steroids in osteoarthritis. Rheumatol Rehabil 1980;19:212-7.

19. Jess D Salinas Jr, MD. Corticosteroid Injections of Joints and Soft Tissues. Web MD; Aug 20, 2014.

20. Ebnezer J, "Osteoarthritis; Viscosuplementation in osteoarthritis knee" Chapter 47 in John Ebnezer Text book of Orthopaedics, $4^{\text {th }}$ edition 2010, pp.681

21. Laurent TC. Fraser R. Hyluronan. FASEB J 1992;6:2397-404.

22. Balazs EA. Denlinger JL. Viscosupplementation: new concept in the treatment of OA. J Rheumatol 1993;20 (supplement 39):3-9.
23. Spelling PF. Heise N. Tolondo OMS: Glycosaminoglycans rheumatoid arthritis. Clin Exp. Rheumatol 1991:9:195-9.

24. Dahl LB. Dahl IMS, Engstrom-Laurent A. Granth K. Concentration and molecular weight of sodium hyluronate in snovial fluid from patients with rheumatoid arthritis and other arthrographies. Ann Rheum Dis 1985;44:817-22.

25. Abatangelo G. O'regan M; Hyluronan: Biological role and function in articular joints. Eur J Rheumatol Inflamm 1995;15:9-16.

26. Arrich J, Piribauer F, Mad P, Schmid D, Klaushofer K, Mullner M. Intra-articular hyaluronic acid for the treatment of osteoarthritis of the knee: systematic review and meta-analysis. CMAJ 2005;172(8):1039-43.

27. Wang CT, Lin J, Chang CJ, Lin YT, Hou SM. Therapeutic effects of Hyaluronic acid on osteoarthritis of the knee. A meta-analysis of randomized Controlled trials. J Bone Joint Surg Am 2004;86-A(3):538-45.

28. Frizziero L. Intra-articular hyaluronic acid in treatment of osteoarthritis of the knee. Clin Exp Rheumatol 1998;16:441-9.

29. Sharma NR, Singh S, Kumar D, Kumar S. Correlation between WOMAC score and hyluronic acid levels in knee Osteoarthritis. Int J Res Med Sci 2015;3:757-62.

30. Smith M.D. Joint lavage and intraarticular steroids in knee OA. J Br Soc Rheumatol 2003;42:1477-1585.

31. Xinning Li. Arthroscopic debridement of the osteoarthritic knee combined with hyaluronic acid treatment. J Orthop Surg Res 2008;3:43.

32. Dervin GF, Stiell IG, Rody K, Grabowski J. Effect of arthroscopic debridement for osteoarthritis of the knee on health-related quality of life. J Bone Joint Surg Am 2003;85:10-9.

How to cite this article: Patel V, Rohra N. A prospective comparative study of functional outcome following arthroscopic debridement and hydrocortisone injection vs arthroscopic debridement and hyaluronicacid injection for the treatment of early primary osteoarthritis knee. Indian $J$ Orthop Surg 2019;5(2):136-40. 